\title{
Ellagic acid produces neuroprotection against LPS- induced dopamine neurotoxicity via the inhibition of microglial NLRP3 inflammasome activation
}

Xue-mei He

Zunyi Medical University

Qiu-yu Yang

Zunyi Medical University

Jing-yi He

Zunyi Medical University

Shuo Sheng

Zunyi Medical University

Jing-jie Li

Zunyi Medical University

Dai-di Li

Zunyi Medical University

Guo-qing Wang

Zunyi Medical University

Feng Zhang ( $\nabla$ zhangfengzmc@163.com )

Zunyi Medical University

\section{Research}

Keywords: Parkinson's disease, Microglia, Inflammasome, Ellagic acid, Neuroprotection

Posted Date: November 27th, 2019

DOI: https://doi.org/10.21203/rs.2.17707/v1

License: (1) (1) This work is licensed under a Creative Commons Attribution 4.0 International License.

Read Full License 


\section{Abstract}

Background: Neuroinflammation plays a crucial role in the pathological process of Parkinson's disease (PD). Nod-like receptor protein 3 (NLRP3) inflammasome was highly located in microglia and involved in the process of neuroinflammation. Activation of NLRP3 inflammasome has been confirmed to contribute to the progression of PD. Thus, inhibition of NLRP3 inflammasomes activation could be an important breakthrough point in PD drug therapy. Ellagic acid (EA) is a natural polyphenol that has been widely found in soft fruits, nuts and other plant tissues with various anti-inflammatory and anti-oxidant properties. However, the mechanisms underlying EA-mediated anti-inflammatory and neuroprotection have not been fully elucidated.

Methods: In this study, lipopolysaccharide (LPS)-induced rat dopamine (DA) neuronal damage model was performed to determine the effects of EA on the protection of DA neurons. Furthermore, DA neuron MN9D cell line and microglia BV-2 cell line were employed to explore whether EA-mediated neuroprotection was through an NLRP3-dependent mechanism.

Results: EA ameliorated LPS-induced DA neuronal loss in rat substantia nigra. Furtherly, inhibition of microglial NLRP3 inflammasome signaling activation was involved in EA-generated neuroprotection, as evidenced by the following observations. First, EA reduced NLRP3 inflammasome signaling activation in microglia and the subsequent pro-inflammatory cytokines excretion. In addition, EA-mediated antineuroinflammation and further DA neuroprotection from LPS-induced neurotoxicity was not shown upon microglial NLRP3 siRNA treatment.

Conclusions: This study demonstrated EA has a profound effect on protecting DA neurons against LPSinduced neurotoxicity via the suppression of microglial NLRP3 inflammasome signaling activation.

\section{Introduction}

Parkinson's disease (PD) is a chronic progressive neurodegenerative disease characterized by a deep selective loss of dopamine (DA) neurons in the substantia nigra (SN) [1]. Clinical manifestations include static tremor, slow movement, postural instability, stiffness and other motor disorders [2]. So far, only a few drugs have been available for PD treatment and most of them just relieve symptoms, not prevent the death of DA neurons [3].

It was recognized that age-related excessive oxidative stress led to DA auto-oxidation, a-synuclein accumulation, and glial cells activation [4], which are the main causes of neuroinflammation [5]. Furtherly, neuroinflammation, characterized by microglia activation and infiltrating $T$ cells at sites of neuronal injury, was considered to be a prominent contributor to the pathogenesis of progressive PD [6]. Microglia are natural immune barriers in the immune system. While stimulated by external conditions, such as brain damage, inflammation and pathogens, microglia could be activated and secrete a range of proinflammatory factors [7]. These factors led to the damage of adjacent DA neurons, which in turn elicited more microglia to reactivate in response, forming a self-propelling cycle [8]. The inflammasome involving 
NLRP3 (NLR family, pyrin domain containing 3)-CASP1 (caspase 1) can regulate the maturation of IL-1 $\beta$ and IL-18 [9, 10]. Additionally, nod-like receptor protein 3 (NLRP3) inflammasome was highly located in microglia and involved in the process of neuroinflammation [11]. Activation of NLRP3 inflammasomes had been discerned to play an important role in the progression of neurodegenerative diseases [12]. Therefore, inhibition of NLRP3 inflammasomes activation could be an important breakthrough point in PD drug therapy.

Ellagic acid (2, 3, 7, 8-tetrahydroxybenzopyrano [5, 4, 3-cde] benzopyran-5-10-dione, EA) is rich abundantly in plant extracts and fruits. EA exhibits a number of pharmacological activities, such as anti-oxidant and anti-inflammatory effects [13]. Recent studies confirmed EA exerted neuroprotection against aging, ischemic stroke and neurodegenerative disorders. For example, EA generated neuroprotection and improved cognitive dysfunctions in sporadic Alzheimer's disease animal model [14]. In addition, EA was indicated to confer neuroprotection against ischemic stroke [15]. However, the underlying mechanisms remain unclear. In this study, rat substantia nigral stereotaxic single injection of lipopolysaccharide (LPS)elicited DA neuronal loss was performed to investigate EA-exerted neuroprotection and the underlying mechanisms as well. These findings would provide evidence for the future application of EA on PD treatment.

\section{Materials And Methods}

\section{Reagents}

EA (purity>95\%), LPS (Escherichia coli 0111:B4) and 6-hydroxydopamine (6-OHDA) were obtained from Sigma Aldrich (St. Lewis, CA, USA). Enzyme-linked Immunosorbant assay (ELISA) kits for TNF-a, IL-1 $\beta$ and IL-18 were bought from Elabscience Biotechnology Co., Ltd (Wuhan, China). MTT assay kit was from Beijing Solarbio science and Technology Co., Ltd. (Beijing, China). The small interfering RNA (siRNA) against NLRP3 was purchased from GenePharma (Shanghai, China). Anti-CR3 complement receptor (OX42 Catalog No. Ab1211) and tyrosine hydroxylase (TH, Catalog No. Ab113) antibodies were bought from Abcam (Cambridge, MA, USA). Anti-caspase-1 (Catalog. No. 22915-1-AP), ionized calcium-binding adapter molecule-1 (Iba-1, Catalog. No. 10904-1-AP), $\beta$-actin (Catalog. No. 20536-1-AP), rabbit IgG (Catalog. No. SA00001-2), and mouse IgG (Catalog. No. SA00001-1) antibodies were purchased from Proteintech Group (Chicago, IL, USA). Anti-NLRP3 (Catalog. No. orb101128) antibody was purchased from Biorbyt (Cambridge, United Kingdom).

\section{Animal and Treatment}

Male Wistar rats (200-250 g) were bought from the Experimental Animal Center in the Third Military Medical University. All experimental procedures were carried out in accordance with Chinese Guidelines of Animal Care and Welfare and this study received an approval from the Animal Care and Use Committee of Zunyi Medical University (Zunyi, China). Rats were acclimated to their environment for 1 week before 
the experiments. All the animals were randomly allocated to five experimental groups with six rats in each group: control, EA alone (50 mg/kg), LPS, LPS+EA (10 mg/kg) and LPS+EA (50 mg/kg). With anesthetized by $7 \%$ chloral hydrate $(0.5 \mathrm{ml} / 100 \mathrm{~g}, \mathrm{v} / \mathrm{w})$, rats received a single LPS (10 $\mu \mathrm{g}$ in $5 \mu \mathrm{l}$ PBS) unilateral injection into the SN pars compacts followed by the coordinates $5.2 \mathrm{~mm}$ posterior to bregma, $1.9 \mathrm{~mm}$ lateral to the midline, and $8.0 \mathrm{~mm}$ ventral to the surface of the skull [16]. After seven daily intragastric administration of EA, rat behavior changes were analyzed by rotarod test. Afterwards, animals were sacrificed and the biochemical analysis was performed.

\section{Rotarod Test}

Rotarod test was performed to study the muscular coordination. It contained cylindrical arrangement of the thin steel rods with two parts by compartmentalization to permit the detection of two rats at the same time. Prior to the test start, all animals were trained to stay at $0 \mathrm{rpm}$ for a while, then steadily increased to $10 \mathrm{rpm}$ in $30 \mathrm{~s}$ and $5 \mathrm{rpm}$ per $30 \mathrm{~s}$ until rats slid off the steps. The duration time each rat stayed on the rod was recorded and calculated for analyzing the behavior changes of rats [17].

\section{Immunohistochemical Analysis and Cell Counting in the SN}

Rat brains were cut with a horizontal sliding microtome into $35 \mu \mathrm{m}$ transverse free-floating sections and immunostained with the corresponding antibodies. DA neurons were recognized with an anti-TH antibody. Then brain slices were incubated with $0.3 \%$ Triton X-100 and closed with goat serum. Subsequently, brain slices were concentrated with anti-TH (1:500), OX-42 (1:800), and NLRP3 (1:800) antibodies at $4{ }^{\circ} \mathrm{C}$ overnight, respectively [18]. Digital images of TH-positive neurons, OX-42-positive microglia and NLRP3-positive inflammasome in midbrain SN were acquired by an Olympus microscope (Olympus, Tokyo, Japan).

\section{Cell Culture and Treatment}

Mouse microglial BV-2 cell lines were obtained from China Center for Type Culture Collection (Wuhan, China). Cultures were maintained in minimum essential medium (MEM) supplemented with $10 \%$ heatinactivated fetal bovine serum (FBS), $100 \mathrm{U} / \mathrm{ml}$ penicillin, and $100 \mu \mathrm{g} / \mathrm{ml}$ streptomycin at $37^{\circ} \mathrm{C}$ in the humidified atmosphere of $5 \% \mathrm{CO}_{2}$ and $95 \%$ air [19]. DA neuron MN9D cell lines were purchased from the Cell Culture Center in the Institute of Basic Medical Sciences of Chinese Academy of Medical Sciences (Beijing, China). MN9D cells were cultured in DMEM medium with $10 \%$ FBS and $1 \%$ penicillinstreptomycin on an atmosphere with $5 \% \mathrm{CO}_{2}$ at $37^{\circ} \mathrm{C}$ in the humidified atmosphere of $5 \% \mathrm{CO}_{2}$ and $95 \%$ air [20]. 


\section{MTT Assay}

Cell viability was evaluated by MTT assay. BV- 2 and MN9D cells were cultured in $1 \times 10^{5} /$ well in 96 -well plates for $24 \mathrm{~h}$. Afterwards, cells were treated with different concentrations of EA for $30 \mathrm{~min}$ followed by LPS $(100 \mathrm{ng} / \mathrm{ml})$ or $6-O H D A(100 \mu \mathrm{M})$ treatment for $24 \mathrm{~h}$ and then incubated with MTT solution $(5 \mathrm{~g} / \mathrm{l})$ for $4 \mathrm{~h}$. Formazan crystals in the cells were solubilized using $200 \mu \mathrm{l}$ dimethyl sulfoxide (DMSO) and the absorbance was detected by an automated microplate reader within $490 \mathrm{~nm}$ wavelength [21].

\section{Western Blot Analysis}

Total protein content was extracted from rat midbrain tissue and BV-2 cells using a lysis buffer containing protease inhibitors. Protein levels were quantified by BCA assay [22]. Protein $(10 \mu \mathrm{g})$ from each sample was subjected to SDS-PAGE gel under the reduced conditions. Proteins were then transferred onto polyvinylidene fluoride (PVDF) membranes. The membranes were blocked with $5 \%$ nonfat milk for $2 \mathrm{~h}$ at room temperature and then incubated overnight at $4{ }^{\circ} \mathrm{C}$ with the primary antibodies: Iba-1, NLRP3, caspase-1, TNF-aIIL-1 $\beta$, IL-18 and $\beta$-actin. Next, the membranes were incubated for $1 \mathrm{~h}$ with a horseradish-peroxidase-conjugated anti-mouse IgG antibody or anti-rabbit IgG at 1:2,000 dilution. The blot films were developed with enhanced ECL Reagent.

\section{ELISA}

The levels of TNF- $a$, IL-1 $\beta$, and IL-18 were measured by ELISA according to the manufacturer's instructions. The microplate reader was used to measure the absorbance at $450 \mathrm{~nm}$ [23].

\section{Immunofluorescence Staining}

Activated microglia were identified with an anti-0X-42 antibody. Cells were fixed with paraformaldehyde (4\%) for $30 \mathrm{~min}$. Later, cells were permeabilized using triton X-100 (0.3\%) for $15 \mathrm{~min}$. Then, cells were blocked using goat serum blocking solution for $60 \mathrm{~min}$ at $37^{\circ} \mathrm{C}$. Thereafter, cells were incubated with 1:800 dilution of anti-OX-42 antibody overnight at $4{ }^{\circ} \mathrm{C}$. Following overnight incubation, cells were incubated in dark for 30 min with goat anti-rabbit secondary antibody (1:1000) or goat anti-mouse secondary antibody (1:1000). Cells were also counterstained with DAPI for 5 min [24]. After rinsing cells with PBS, representative fluorescence images were obtained using EVOS ${ }^{\circledR}$ Floid® Cell imaging station. 


\section{RNA Transfection}

BV2 cells were cultured and seeded in a 6-well plate at a density of $1 \times 10^{5} \mathrm{cells} / \mathrm{ml}$. The transfection of siRNA was performed complying with the manufacturer's protocol. GP-siRNA-Mate plus was used to transfect with siRNAs. After $6 \mathrm{~h}$ of transfection, the transfection solution was removed and cells were rinsed with PBS and replaced with MEM medium containing 2\% FBS followed by treatment of EA and/or LPS [25].

\section{Statistical Analysis}

Results were indicated as mean \pm standard error of the mean (SEM). Statistical significance was analyzed by one-way analysis of variance (ANOVA) using GraphPad Prism software (GraphPad Software Inc., San Diego, CA, USA). Upon ANOVA demonstrating the significant differences, pairwise comparison between means was evaluated by Bonferroni's post hoc test with correction. A value of $p<0.05$ was considered statistically significant.

\section{Results}

EA attenuated LPS-induced DA neuronal damage in the $S N$ in vivo

Neuroprotective effects of EA on LPS-induced DA neuronal damage were investigated in rats. As shown in Fig. 1A, LPS reduced the time rat stayed on the rod, compared with control group. However, EA attenuated LPS-caused decrease in the time rat remained on the rod. To further confirm EA-mediated DA neuroprotection, $\mathrm{TH}$-positive neuronal number and TH protein expression were determined. As shown in Fig. 1B, EA ameliorated LPS-induced decrease in TH protein expression. Consistent with TH protein detection results, TH-positive neuronal counting showed EA protected against LPS-induced DA neuronal loss (Fig. 1C).

EA ameliorated LPS-elicited activation of microglia and NLRP3 inflammasome signaling in vivo

Next, the effects of EA on microglia and NLRP3 inflammasome activation were investigated. To verify the connection between NLRP3 inflammasome and microglia, the immunofluorescence double calibration site was conducted. As shown in Fig. 2A, the NLRP3 inflammasome was activated and located in activated microglia. EA attenuated LPS-induced activation of NLRP3 inflammasome in microglia. Also, EA inhibited Iba-1 protein expression induced by LPS (Fig. 2B). In addition, EA suppressed LPS-induced activation of NLRP3 inflammasome signaling (Fig. 2C) and pro-inflammatory cytokines (1L-1 $\beta$, TNF-a and 1L-18) protein expressions (Fig. 2D).

EA had no direct neuroprotective effects on DA neurons 
To further confirm whether EA produced direct neuroprotective actions on DA neurons, the effects of EA on 6-OHDA -induced DA neuronal damage in vitro were determined. First, as shown in Fig. 3A, 6-OHDA induced neurotoxicity was not attenuated by EA treatment in MN9D cell-enriched cultures. Next, in THpositive neurons counting analysis (Fig. 3B and C), 6-OHDA caused TH-positive neuronal loss and EA didn't protect from 6-OHDA-induced neuronal damage. Similar results were indicated in TH protein detection shown in Fig. 3D. These results demonstrated that EA didn't generate direct neuroprotection on DA neurons.

\section{EA inhibited microglial NLRP3 inflammasome activation in vitro}

The effects of EA on microglia and NLRP3 inflammasome signaling activation were further confirmed in vitro. First, BV-2 cells were employed to examine the effects of EA on LPS-induced microglial activation. As shown in Fig. 4A, immunofluorescence staining assay indicated that EA reduced LPS-induced microglia activation. Also, EA decreased LPS-induced higher protein expression of Iba-1 (Fig. 4B). Then, EA inhibited the activation of microglial NLRP3 inflammasome signaling induced by LPS (Fig. 4B). In addition, EA eliminated LPS-induced production of pro-inflammatory factors, such as TNF-a, IL-1 $\beta$ and IL18 in the culture medium (Fig. 4C).

NLRP3 inflammasome signaling inactivation was involved in EA-mediated anti-inflammatory properties

To investigate the role of NLRP3 inflammasome signaling in EA-mediated anti-neuroinflammation, NLRP3 siRNA was performed in BV-2 cell cultures. First, as shown in Fig. 5A, NLRP3 siRNA was transfected into microglia cells and the successful transfection with NLRP3 siRNA was evaluated by NLRP3 protein level in BV-2 cells. Furthermore, NLRP3 siRNA and EA inhibited LPS-induced NLRP3 inflammasome signaling activation, respectively. However, no significant difference of NLRP3, caspase-1 and pro-caspase-1 protein expressions between LPS + EA and LPS + EA + NLRP3 siRNA groups was discerned (Fig. 5B). In addition, the effects of EA on pro-inflammatory factors excretion with NLRP3 siRNA administration were measured. In parallel with NLRP3 inflammasome signaling analysis, EA didn't reduce LPS-induced release of pro-inflammatory factors again after NLRP3 siRNA treatment (Fig. 5C). These observations indicated EA attenuated microglia-induced neuroinflammation via the inhibition of NLRP3 inflammasome signaling activation.

\section{EA targeted microglial NLRP3 inflammasome to produce DA neuroprotection}

Since microglia were the target of EA-generated DA neuroprotection, whether this neuroprotection resulted from inhibiting microglial NLRP3 inflammasome activation was then explored. As shown in Fig. 6, compared with MCM (LPS) group, both MCM (LPS + NLRP3 siRNA) and MCM (LPS + EA) protected against MCM (LPS)-induced neurotoxicity evidenced by cell viability and TH protein expression detection, whereas no significant difference of neuroprotection between these two groups was exhibited. Furtherly, MCM (LPS + NLRP3 siRNA + EA) didn't exert more DA neuroprotection against MCM (LPS)-caused neuronal injury than MCM (LPS + NLRP3 siRNA) or MCM (LPS + EA) treatment, either. 


\section{Discussion}

The present study aimed to investigate the neuroprotective actions of EA on LPS-induced DA neuronal loss and evaluate the role of microglia in this neuroprotection. Results indicated that EA protected DA neurons against LPS-induced neurotoxicity in SN. Furtherly, inhibition of microglial NLRP3 inflammasome signaling activation was involved in EA-generated neuroprotection, as evidenced by the following observations. First, EA reduced NLRP3 inflammasome signaling activation in microglia and the subsequent pro-inflammatory cytokines excretion. In addition, EA-mediated anti-neuroinflammation and further DA neuroprotection from LPS-induced neurotoxicity was not shown upon microglial NLRP3 siRNA treatment. Taken together, EA conferred neuroprotection against LPS-induced DA neuronal damage via the inhibition of microglial NLRP3 inflammasome signaling activation.

Neuroinflammation is considered to be a most common feature of the aging brain and neurodegenerative disorders including PD. It is primarily mediated by activated glial cells and accompanied by the excretion of pro-inflammatory mediators [26]. As the first defense of immune surveillance, microglia readily become activated and predominately participate in inflammatory response. In addition, astroglia could amplify microglia-mediated neuroinflammation and result in the feedback loop of neuroinflammatory reactions [27]. In this regard, understanding the molecular mechanisms of PD can be found by investigating the microglial neuroinflammation [28]. Therefore, to target microglia-derived pro-inflammatory cytokines might offer promising therapeutic approaches for PD management. Inflammasomes are multi-protein complexes responsible for intracellular sensors of environmental and cellular stress [29]. Inflammasomes consist of NLRP family, the signaling adapter apoptosis associated speck-like protein containing a caspase recruitment domain (ASC) and pro-inflammatory precursor pro-caspase-1 [30]. In addition, NLRP3 inflammasome is particularly located in microglia and involved in the response of neuroinflammation [11]. Upon cellular stress, assembly of NLRP3 inflammasome triggers caspase-1 activation and further caspase-1-mediated production of IL-1 $\beta$ and IL-18, thereby initiating neuroinflammation [31]. In PD patient brains, the NLRP3 inflammasome is potentially activated by insoluble a-synuclein aggregates and oxidative stress [32]. However, deficiency of NLRP3 inflammasome attenuates motor dysfunction and DA neurodegeneration in PD mouse model [33]. Thus, inhibition of NLRP3 inflammasome activation might be beneficial for PD intervention. In the present study, EAmediated DA neuroprotection was discerned in neuron-microglia co-cultures but not in neuron-enriched cultures, implying that microglia were at least essential for EA-generated neuroprotection. Moreover, we found EA reduced pro-inflammatory factors production induced by LPS. Furtherly, EA-inhibited microglial activation and subsequent pro-inflammatory factors release was attributed to the inhibition of microglial NLRP3 inflammasome activation. This finding was revealed by the following observations: 1) EA inhibited microglial NLRP3 and pro-caspase-1 activation and IL-1 $\beta$ production. 2) EA could not further suppress LPS-induced pro- inflammatory factors release and produce DA neuroprotection after neuronmicroglia co-cultures treated by NLRP3 siRNA.

To date, current PD therapy is focused on the symptoms control and fails to delay the progressive neurodegenerative process. Actually, various side-effects of the available drugs present huge challenges 
for long-term application. Therefore, more potential therapeutic candidates are urgently essential for halting the progression of PD. Recent studies demonstrated inhibition of neuroinflammation would attenuate DA neurodegeneration. Thus, anti-inflammatory agents might open new avenues for PD treatment. However, the low success of translating promising anti-inflammatory candidates from animal studies to clinical trials was indicated. Therefore, an urgent approach for novel anti-inflammatory alternatives design was prompted [29]. Here, the present study demonstrated that EA protected from LPSinduced DA neurotoxicity and modulation of microglial NLRP3 inflammasome signaling activation was revealed to participate in this neuroprotection. However, the current study on EA only stays in the animal experiments, and the follow-up hopes to be used as a clinical drug application for the next research.

\section{Conclusions}

This study demonstrated EA has a profound effect on protecting DA neurons against LPS-induced neurotoxicity via the suppression of microglial NLRP3 inflammasome signaling activation. These findings suggest EA might be a potential benefit for PD treatment.

\section{Abbreviations}

6-OHDA: 6-hydroxydopamine; AD: Alzheimer's disease; PD: Parkinson's disease; SN: Substantia nigra; DA: Dopamine; LPS: Lipopolysaccharide; NLRP3: Nod-like receptor protein 3; CASP1: Cysteinyl aspartatespecific proteases-1; EA: Ellagic acid; FBS: Fetal bovine serum; MEM: Minimum essential medium; IL-1 $\beta$ : Interleukin-1 $\beta$; IL-18: Interleukin-18; TNF-a: Tumor necrosis factor-a; ELISA: Enzyme-linked Immunosorbant assay; siRNA: Small interfering RNA; OX-42: Anti-CR3 complement receptor; PBS: phosphate buffer; DMSO: dimethyl sulfoxide; PVDF: polyvinylidene fluoride; Iba-1: Ionized calcium-binding adapter molecule-1; TH: Tyrosine hydroxylase; MCM: Microglia-conditioned Medium

\section{Declarations}

\section{Acknowledgements}

Not applicable

\section{Funding}

This study was supported by the National Natural Science Foundation of China (No. 81760658), the foundation for High-level Innovative Talents of Guizhou Province (No. 20164027), Shijingshan's Tutor Studio of Pharmacology (No. GZS-201607), the Innovation Research Group project of Education Department of Guizhou Province (No. 2016038), the foundation for Excellent Young Talents of Zunyi Medical University (No.201603) and Master Start Foundation of Zunyi Medical University (No. F-898). 


\section{Availability of data and materials}

The datasets used and/or analyzed during the current study were available from the corresponding author on the reasonable request.

\section{Authors' contributions}

FZ conceived and designed the experiments. All the authors participated in the experiment performance and data analysis. FZ, SS and XMH wrote, revised and checked the article. All authors revised and approved the final manuscript.

\section{Ethics approval and consent to participate}

All experimental procedures were carried out in accordance with Chinese Guidelines of Animal Care and Welfare and this study received an approval from the Animal Care and Use Committee of Zunyi Medical University (Zunyi, China).

\section{Consent for publication}

Not applicable.

\section{Competing interests}

The authors declared no conflicts of interests.

\section{Publisher's Note}

Springer Nature remains neutral with regard to jurisdictional claims in published maps and institutional affiliations.

\section{References}

1. Zhang, Y., V.L. Dawson, and T.M. Dawson, Oxidative stress and genetics in the pathogenesis of Parkinson's disease. Neurobiol Dis. 2000;7:240-50. 
2. Collier, T.J., N.M. Kanaan, and J.H. Kordower, Aging and Parkinson's disease: Different sides of the same coin? Mov Disord. 2017;32:983-990.

3. Lees, A.J., J. Hardy, and T. Revesz, Parkinson's disease. Lancet. 2009;373:2055-66.

4. Raichur, A., S. Vali, and F. Gorin, Dynamic modeling of alpha-synuclein aggregation for the sporadic and genetic forms of Parkinson's disease. Neuroscience. 2006;142:859-70.

5. Qian, L., P.M. Flood, and J.S. Hong, Neuroinflammation is a key player in Parkinson's disease and a prime target for therapy. J Neural Transm (Vienna). 2010;117:971-9.

6. Hansson, E., Long-term pain, neuroinflammation and glial activation. Scand J Pain. 2010;1:67-72.

7. Zhang, F., J. Liu, and J.S. Shi, Anti-inflammatory activities of resveratrol in the brain: role of resveratrol in microglial activation. Eur J Pharmacol. 2010;636:1-7.

8. Dauer, W. and S. Przedborski, Parkinson's disease: mechanisms and models. Neuron. 2003;39:889909.

9. Franchi, L., R. Munoz-Planillo, and G. Nunez, Sensing and reacting to microbes through the inflammasomes. Nat Immunol. 2012;13:325-32.

10. He, Y., H. Hara, and G. Nunez, Mechanism and Regulation of NLRP3 Inflammasome Activation. Trends Biochem Sci. 2016;41:1012-1021.

11. Heneka, M.T., et al., NLRP3 is activated in Alzheimer's disease and contributes to pathology in APP/PS1 mice. Nature. 2013;493:674-8.

12. Freeman, L.C. and J.P. Ting, The pathogenic role of the inflammasome in neurodegenerative diseases. J Neurochem. 2016;136 Suppl 1:29-38.

13. Firdaus, F., et al., Ellagic acid attenuates arsenic induced neuro-inflammation and mitochondrial dysfunction associated apoptosis. Toxicol Rep. 2018;5:411-417.

14. Moura, F.A., et al., Lipoic Acid: its antioxidant and anti-inflammatory role and clinical applications. Curr Top Med Chem. 2015;15:458-83.

15. Liu, Q.S., et al., Ellagic acid protects against neuron damage in ischemic stroke through regulating the ratio of Bcl-2/Bax expression. Appl Physiol Nutr Metab. 2017;42:855-860.

16. Latchoumycandane, C., et al., Dopaminergic neurotoxicant 6-OHDA induces oxidative damage through proteolytic activation of PKCdelta in cell culture and animal models of Parkinson's disease. Toxicol Appl Pharmacol. 2011;256:314-23.

17. Wang, T., et al., Hydroxysafflor Yellow A Improves Motor Dysfunction in the Rotenone-Induced Mice Model of Parkinson's Disease. Neurochem Res. 2017;42:1325-1332.

18. Zhang, F., et al., Resveratrol protects dopamine neurons against lipopolysaccharide-induced neurotoxicity through its anti-inflammatory actions. Mol Pharmacol. 2010;78:466-77.

19. Song, X.M., et al., [Inhibitory effects of acteoside on LPS-induced inflammatory response on BV-2 microglial cells]. Zhongguo Zhong Yao Za Zhi. 2016; 41:2506-2510.

20. Dong, Y., et al., Amperometric measurements of catecholamine release from single vesicles in MN9D cells. J Neurochem. 2008;107:1589-95. 
21. van Meerloo, J., G.J. Kaspers, and J. Cloos, Cell sensitivity assays: the MTT assay. Methods Mol Biol. 2011;731:237-45.

22. Hirano, S., Western blot analysis. Methods Mol Biol. 2012;926:87-97.

23. Zheng, Y., et al., Icariin targets Nrf2 signaling to inhibit microglia-mediated neuroinflammation. Int Immunopharmacol. 2019;73:304-311.

24. Donaldson, J.G., Immunofluorescence Staining. Curr Protoc Cell Biol. 2015;69:4.3.1-7.

25. Chen, C., et al., Naringenin Produces Neuroprotection Against LPS-Induced Dopamine Neurotoxicity via the Inhibition of Microglial NLRP3 Inflammasome Activation. Front Immunol. 2019;10:936.

26. Shao, W., et al., Suppression of neuroinflammation by astrocytic dopamine D2 receptors via alphaBcrystallin. Nature. 2013;494:90-94.

27. Jo, M., et al., Astrocytic Orosomucoid-2 Modulates Microglial Activation and Neuroinflammation. J Neurosci. 2017;37:2878-2894.

28. Wang, G.Q., et al., Icariin Reduces Dopaminergic Neuronal Loss and Microglia-Mediated Inflammation in Vivo and in Vitro. Front Mol Neurosci. 2017;10:441.

29. Yan, Y., et al., Dopamine controls systemic inflammation through inhibition of NLRP3 inflammasome. Cell. 2015;160:62-73.

30. Hu, Z.L., et al., Kir6.1/K-ATP channel on astrocytes protects against dopaminergic neurodegeneration in the MPTP mouse model of Parkinson's disease via promoting mitophagy. Brain Behav Immun. 2019;81:509-522.

31. Di Virgilio, F., The therapeutic potential of modifying inflammasomes and NOD-like receptors. Pharmacol Rev. 2013;65:872-905.

32. Gordon, R., et al., Inflammasome inhibition prevents alpha-synuclein pathology and dopaminergic neurodegeneration in mice. Sci Transl Med. 2018;10.

33. Lee, E., et al., MPTP-driven NLRP3 inflammasome activation in microglia plays a central role in dopaminergic neurodegeneration. Cell Death Differ. 2019;26:213-228.

\section{Figures}


A

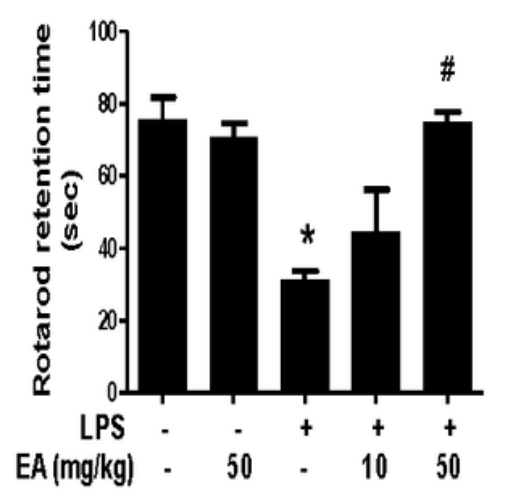

B
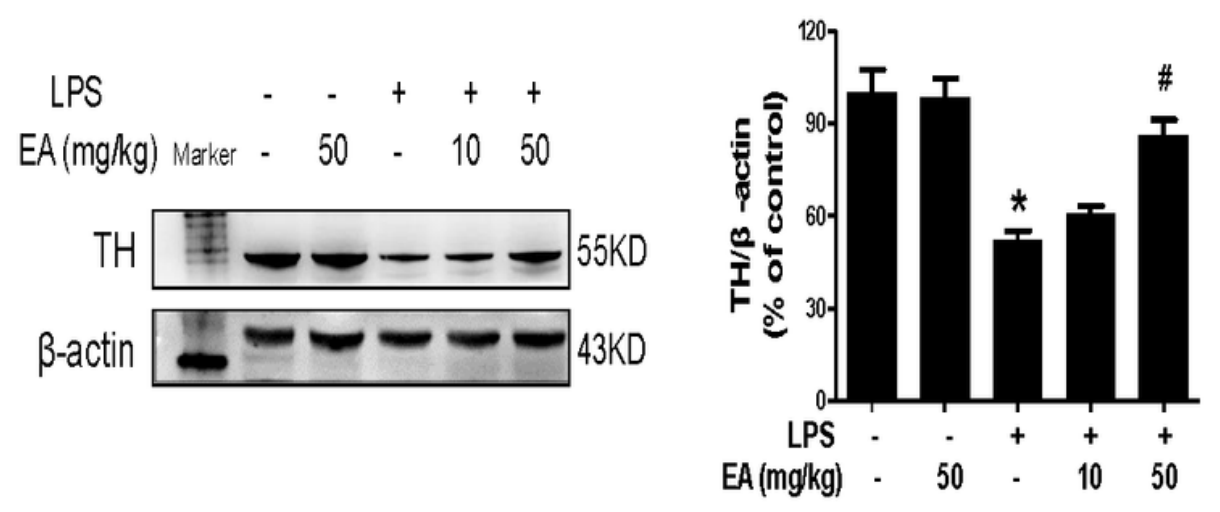

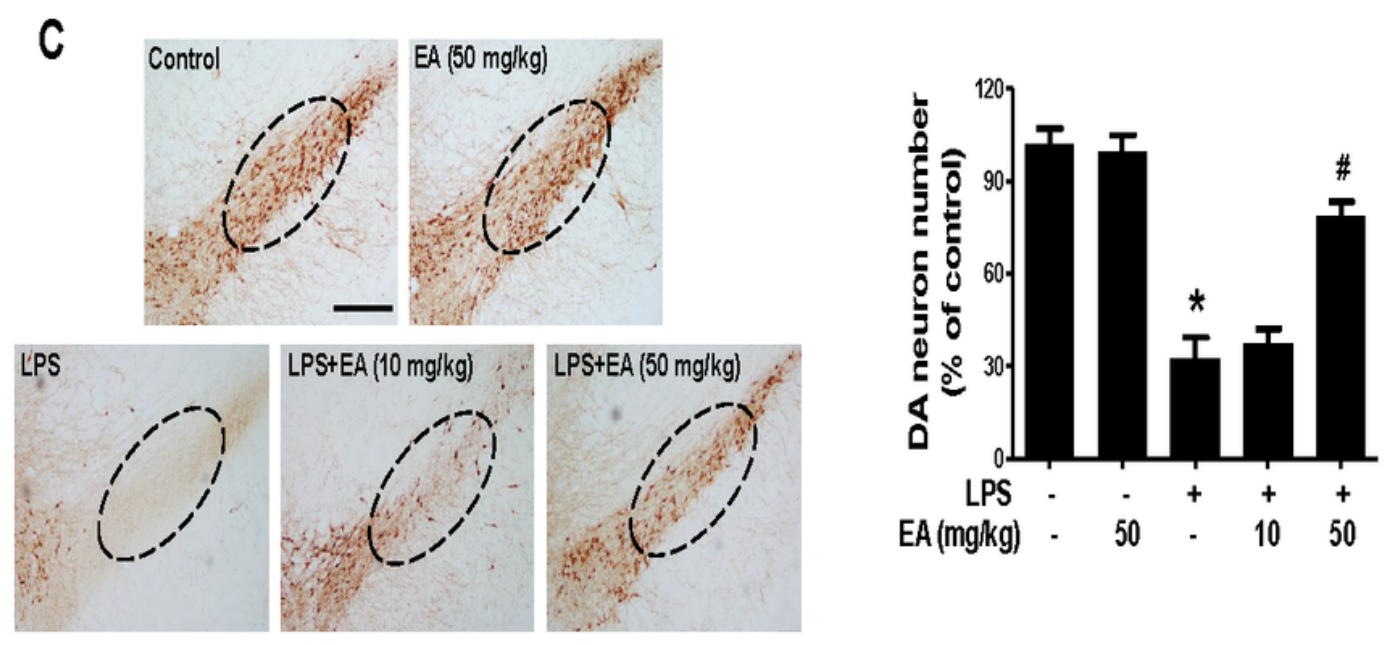

Figure 1

Figure 1

EA attenuated LPS-induced DA neuronal damage in the $S N$ in vivo. Rats were intragastrically given EA ( $50 \mathrm{mg} / \mathrm{kg}$ ) for 7 consecutive days. Rat behavior changes were analyzed by rotarod test (A). TH protein expression in rat midbrain was tested by western blot assay (B). Brain sections were immunostained with an anti-TH antibody (C), and the number of TH-positive neurons in the SN was counted. The "ellipse" presented the area of SN. Scale bar $=200 \mu \mathrm{m}$. Data were mean \pm SEM from 6 mice. ${ }^{*} p<0.05$ compared with control group; \#p<0.05 compared with LPS group. 

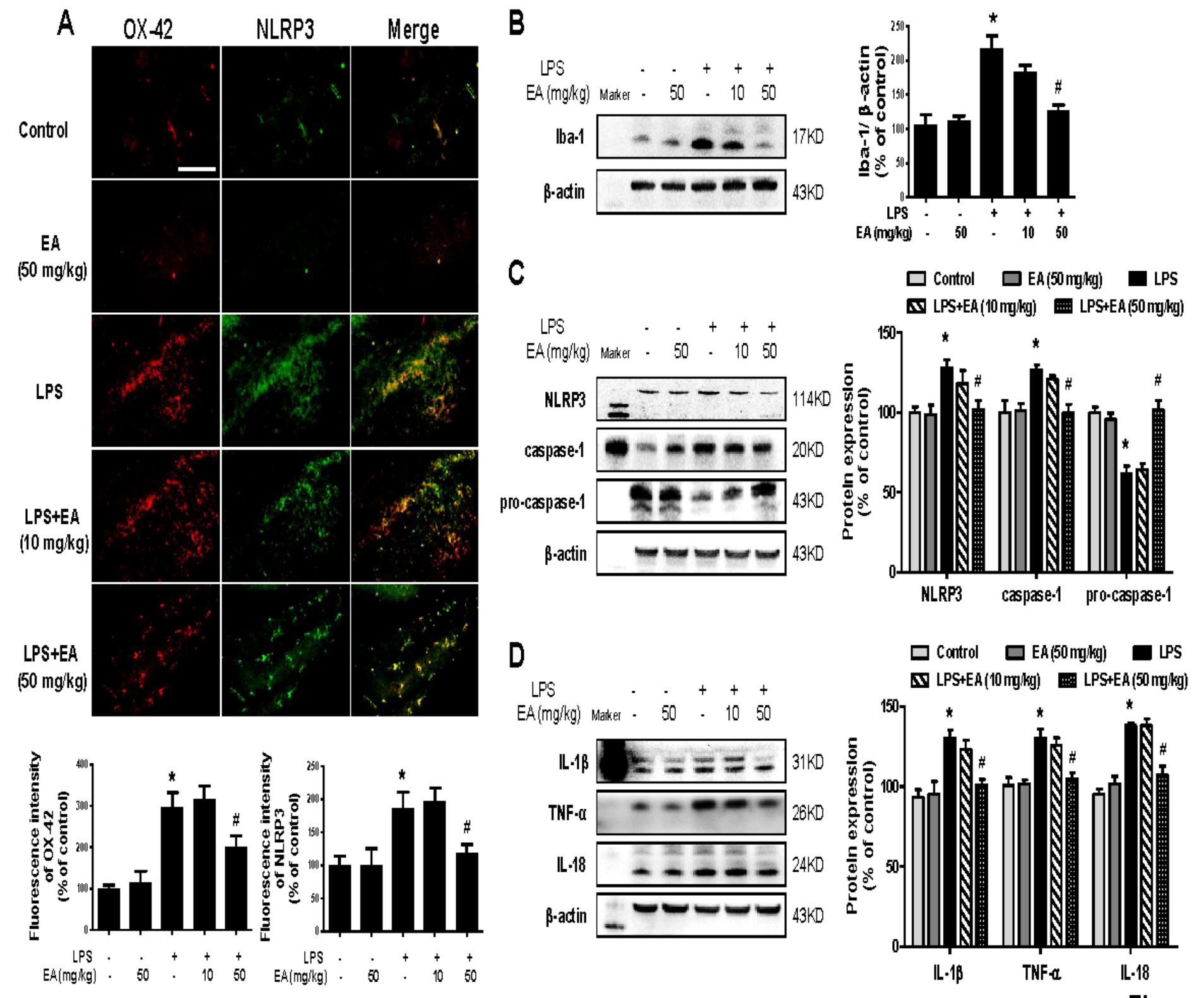

Figure 2

Figure 2

EA ameliorated LPS-elicited activation of microglia and NLRP3 inflammasome signaling in vivo. Rat brains were collected and stained by double-immunofluorescence with anti-NLRP3 and anti-OX-42 antibodies (green fluorescence represented NLRP3 inflammasome and red fluorescence represented microglia) (A). The protein expressions of Iba-1 (B), NLRP3, caspase-1 and pro-caspase-1 (C) and TNF-a, IL-1 $\beta$ and IL-18 (D) in rat midbrain were determined via western blot assay. Data were the mean \pm SEM from 6 rats and expressed as a percentage of the control group. ${ }^{*} p<0.05$ compared with control group; $\# p<0.05$ compared with LPS group. 
A
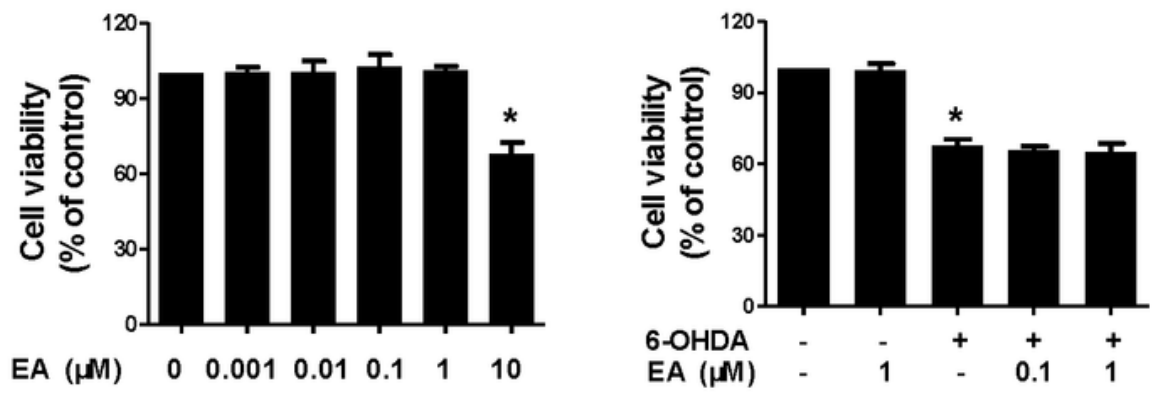

B

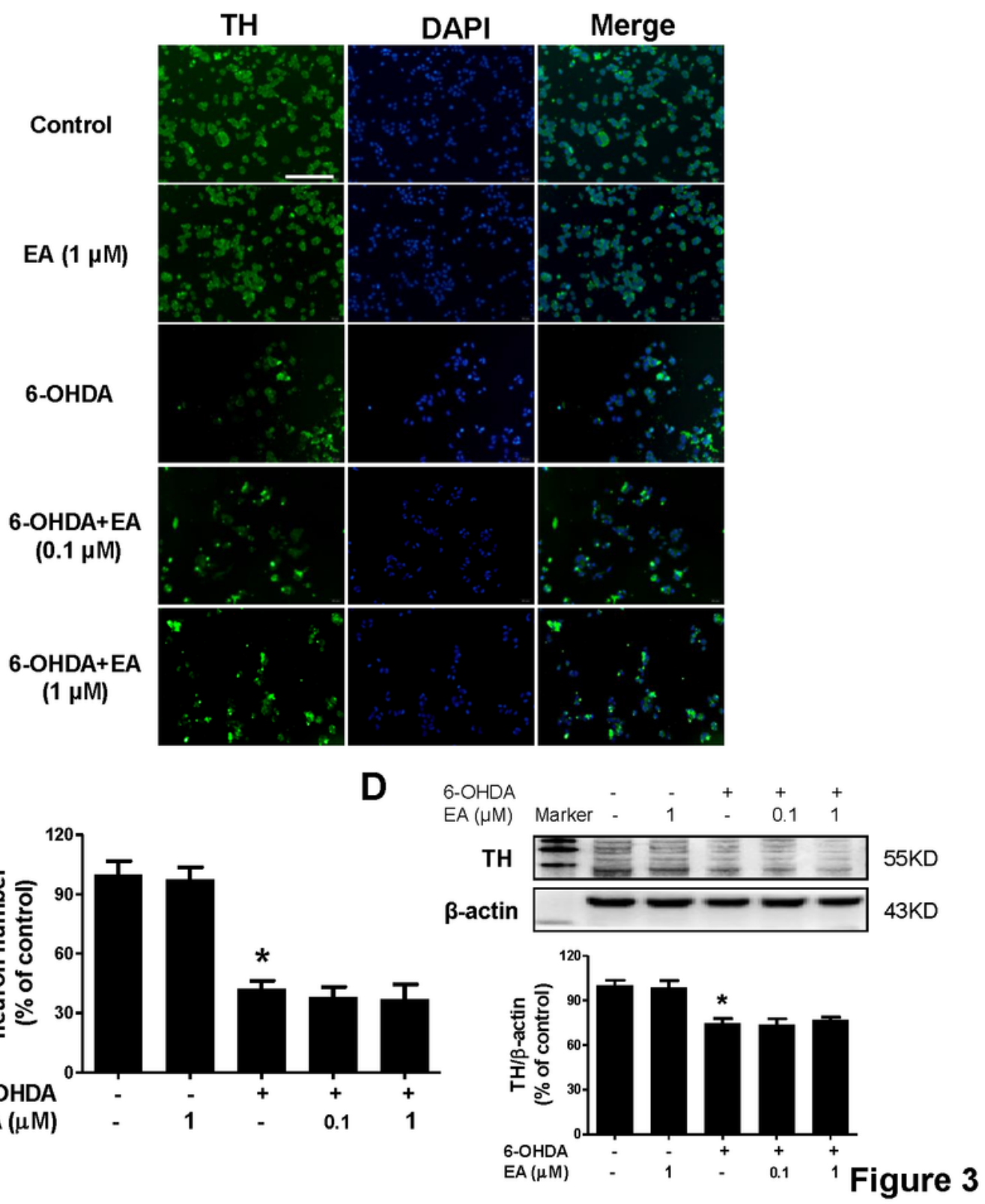

Figure 3

EA had no direct neuroprotective effects on DA neurons. MN9D cells were treated with EA ( 0.1 and $1 \mu \mathrm{M})$ for $30 \mathrm{~min}$ and then incubated with 6-OHDA $(100 \mu \mathrm{M})$ for $24 \mathrm{~h}$. Cell viability was determined by MTT assay (A). 6-OHDA-induced MN9D cell damage was evaluated by immunostaining (B) and cell counting (C). Scale bar $=100 \mu \mathrm{m}$. The protein expression of TH was detected by western blot assay (C). Data were 
the mean \pm SEM from three independent experiments performed in triplicate. ${ }^{*} p<0.05$ compared with control cultures; \#p<0.05 compared with 6-OHDA-treated cultures.
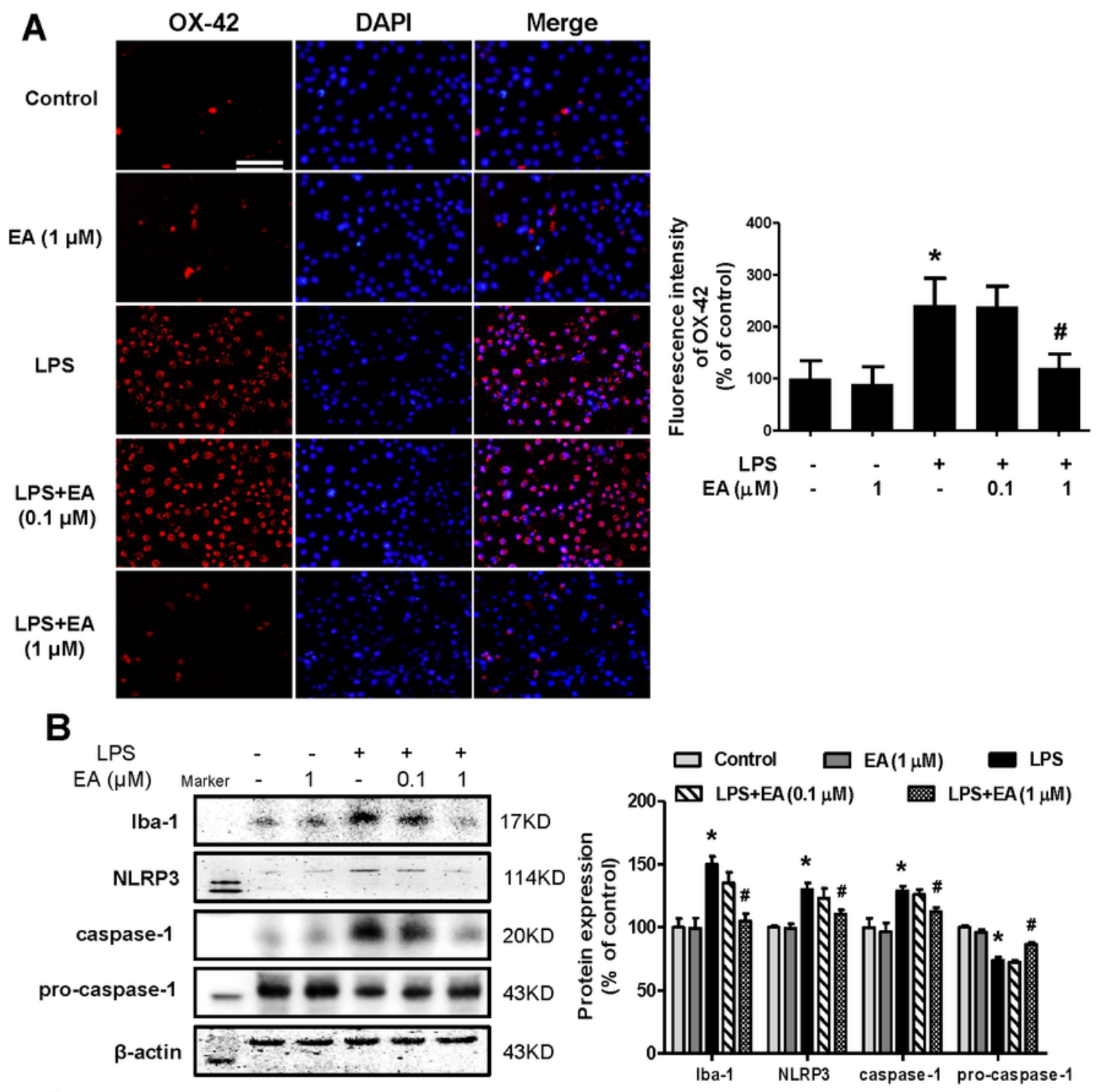

C
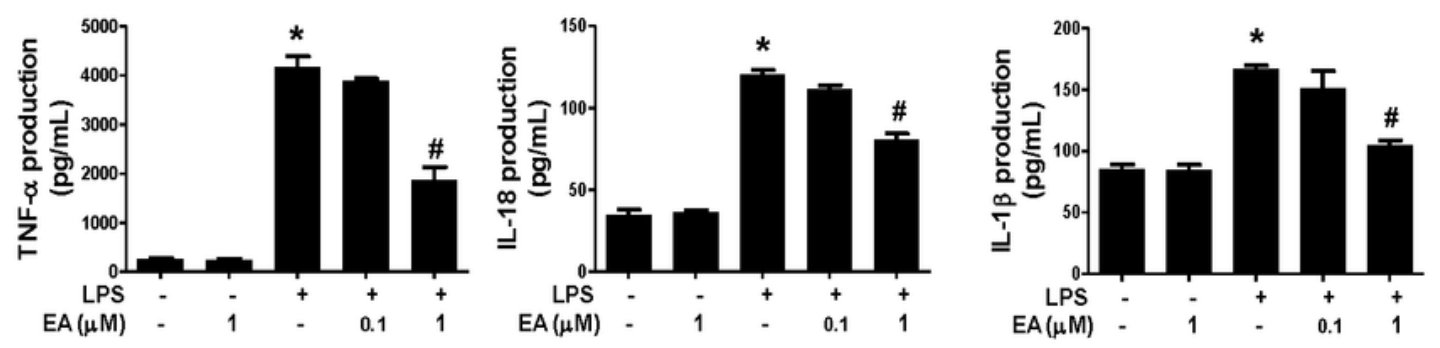

Figure 4

\section{Figure 4}

EA inhibited microglial NLRP3 inflammasome activation in vitro. BV2 cells were treated with EA (0.1 and $1 \mu \mathrm{M})$ for $30 \mathrm{~min}$ and then incubated with LPS $(100 \mathrm{ng} / \mathrm{ml})$ for $24 \mathrm{~h}$. Microglial activation was evaluated by immunostaining (A) with an anti-OX-42 antibody and quantitated by western blot analysis with an 
anti-lba-1 antibody (B). Scale bar $=100 \mu \mathrm{m}$. The effects of EA on NLRP3 inflammasome signaling activation in BV-2 cells were detected via western blotting (B). The ratio of densitometry values of Iba-1, NLRP3, caspase-1 and pro-caspase-1 with $\beta$-actin were analyzed and normalized to each respective control cultures. The release of pro-inflammatory factors, such as TNF-a囚IL-1 $\beta$, and IL-18, in BV-2 cell culture medium was measured by ELISA (C). Data were the mean \pm SEM from three independent experiments performed in triplicate. ${ }^{*} p<0.05$ compared with control cultures; $\# p<0.05$ compared with LPStreated cultures.

A
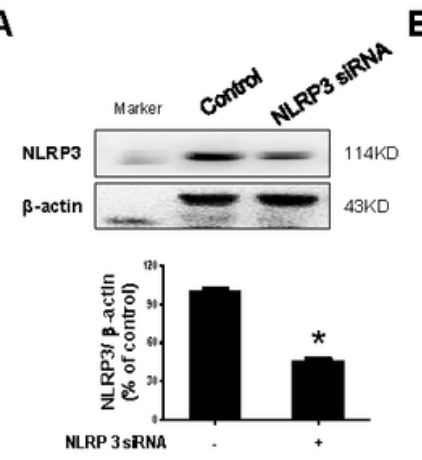

C

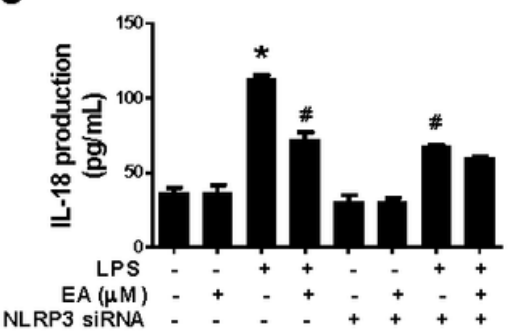

B
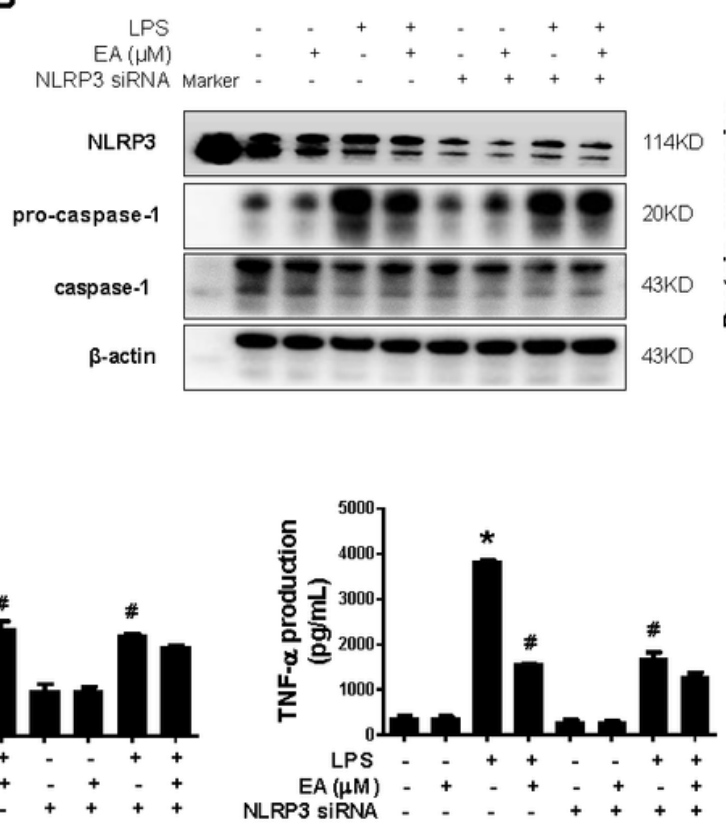
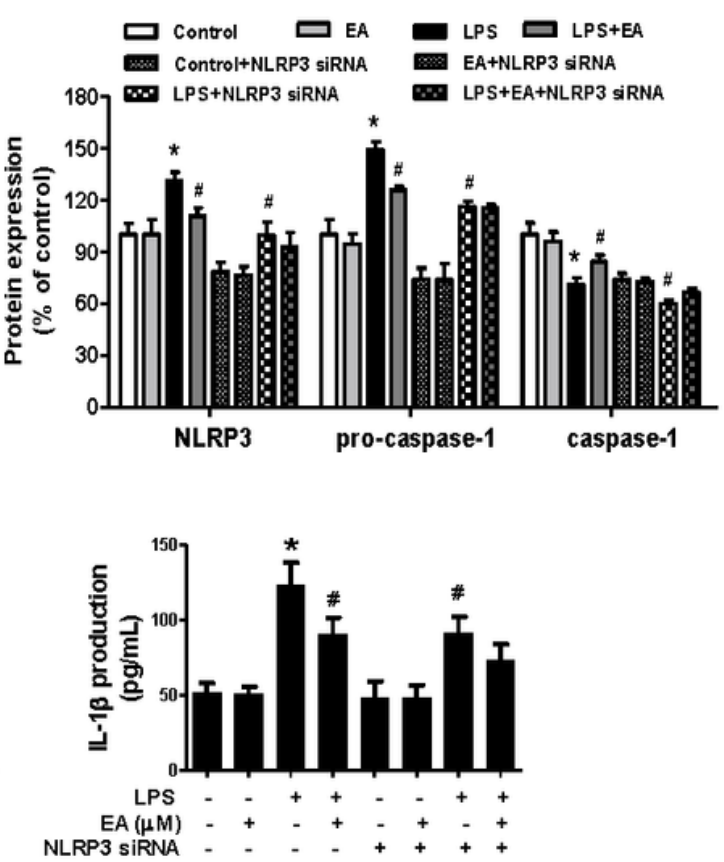

Figure 5

Figure 5

NLRP3 inflammasome signaling inactivation was involved in EA-mediated anti-inflammatory properties. BV-2 cells were treated with NLRP3 siRNA ( $40 \mathrm{nmol} / \mathrm{L})$. After $6 \mathrm{~h}$ of transfection, the transfection solution was removed and cells were rinsed with PBS. The silence efficiency was assessed via NLRP3 protein expression detection (A). Moreover, BV-2 cells were treated with EA $(1 \mu \mathrm{M})$ in the presence of NLRP3siRNA and then exposed to LPS for $24 \mathrm{~h}$. The protein expressions of Iba-1, NLRP3, caspase- 1 and procaspase- 1 in BV-2 cells were detected via western blot assay (B). The levels of TNF- $\alpha$, IL-1 $\beta$ and IL-18 in culture medium were measured by ELISA (C). Data were the mean \pm SEM from three independent experiments performed in triplicate. ${ }^{*} p<0.05$ compared with control cultures; $\# p<0.05$ compared with LPStreated cultures. 


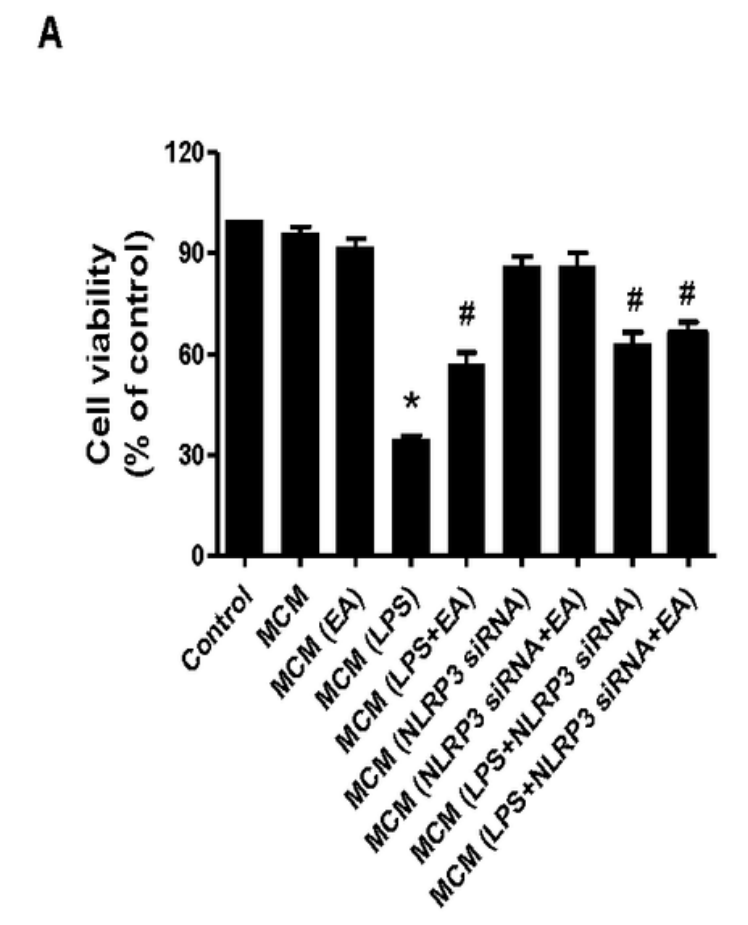

B
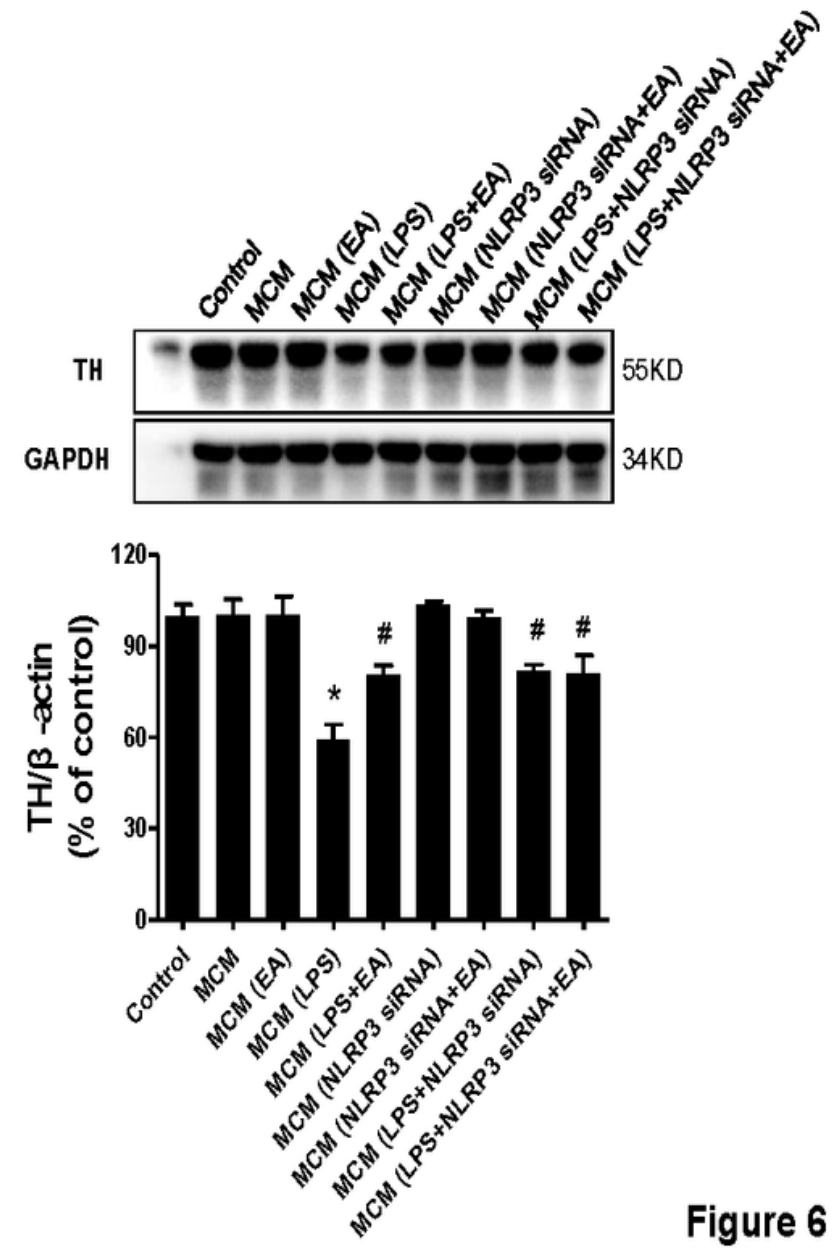

Figure 6

Figure 6

EA targeted microglial NLRP3 inflammasome to produce DA neuroprotection. Microglia-conditioned medium (MCM) prepared from BV-2 cell cultures with administration of EA [MCM (EA)], LPS [MCM (LPS)], LPS + EA [MCM (LPS + EA)], NLRP3 siRNA [MCM (NLRP3 siRNA)], NLRP3 siRNA + EA [MCM (NLRP3 siRNA +EA)], NLRP3 siRNA + LPS [MCM (NLRP3 siRNA + LPS)] , LPS + NLRP3 siRNA + EA [MCM (LPS + NLRP3 siRNA + EA)] were harvested and added to MN9D cells incubated for $24 \mathrm{~h}$. MN9D cell viability was determined by MTT assay (A). TH protein expression was tested by western blot assay (B). Data were the mean \pm SEM from three independent experiments performed in triplicate. ${ }^{*} p<0.05$ compared with control cultures; \#p<0.05 compared with MCM (LPS)-treated cultures. 